Europhys. Lett., 73 (6), pp. 864-870 (2006)

DOI: $10.1209 / \mathrm{epl} / \mathrm{i} 2005-10468-4$

\title{
Localisation-delocalisation transition of electrolytes between micro-electrodes
}

\author{
A. Esztermann, R. Messina and H. Löwen \\ Institut für Theoretische Physik II, Heinrich-Heine-Universität Düsseldorf \\ Universitätsstraße 1, D-40225 Düsseldorf, Germany
}

received 20 October 2005; accepted in final form 17 January 2006

published online 1 February 2006

PACS. 61.20.Qg - Structure of associated liquids: electrolytes, molten salts, etc. PACS. 61.20.Ja - Computer simulation of liquid structure.

PACS. 82.70.Dd - Colloids.

\begin{abstract}
Employing computer simulations and Poisson-Boltzmann theory, we show that a symmetric electrolyte in an electric condenser undergoes a localisation-delocalisation transition as a function of the external voltage $U$. The transition occurs at the critical voltage $U_{\text {c }}$ corresponding to the situation where the surface charge density of the plates equals the area charge density of completely charge-separated electrolytes. The average distance of the electrolyte ions to the plates diverges logarithmically for $U \searrow U_{\mathrm{c}}$. This transition is expected to be observable in micro-electrodes.
\end{abstract}

If a charged body is placed into a salt solution, the cations and anions of the salt will screen the body charge. For high temperatures and/or low charge densities, the traditional linearised screening theory leads to an electric double layer around the charged-body surface which involves an exponentially decreasing density field of the micro-ions as a function of their distance to the surface. One of the simplest set-ups are two parallel homogeneously charged plates with the famous solution of Gouy-Chapman almost a century ago [1,2]. Subsequently, further linear and nonlinear screening theories have been performed and the electrostatic forces acting between two parallel plates have been calculated, see e.g. refs. [3-7] and references therein. Furthermore, the dynamics of density distributions around one charged plate [8] and in a charged disordered background [9] have been investigated.

While the colloidal community has spent much effort to investigate the interaction between like-charge plates which directly affects colloidal stability, one of the central topics of electro-chemistry concerns electrolytes between plates of opposite charges. The latter situation, an electrolyte in a capacitor or condenser, is relevant for a fundamental understanding of electrodes. Here the surface charge density on, or equivalently, the electric field between the plates can be tuned by the external voltage $U$ applied. Recent progress has also been achieved in miniaturising capacitors leading to well-controlled micro-electrodes [10-12] or even nanoelectrodes $[13,14]$ in an electrolyte solution. These are used as basic switching elements in microfluidic devices and in nanoelectronics.

In this letter, we show that symmetric electrolytes in a condenser of two oppositely charged plates undergo a transition from a localised to a delocalised state. The transition occurs as 
a function of external voltage $U$ applied for fixed electrolyte area density and temperature in the limit where the plate distance $D$ goes formally to infinity. The order parameter of the transition is the first moment $h$ of the micro-ion density profiles which is the averaged distance of the anions and cations to the oppositely charged plate. If $\sigma$ denotes the modulus of the charge of anions (or cations) between the plates per unit area, the transition occurs exactly at that voltage $U_{\mathrm{c}}$ which equals the surface charge density of the plates, $\sigma_{\mathrm{p}}$, to $\sigma$. The fingerprint of this transition is a logarithmic divergence of the order parameter $h$ in $\left(U-U_{\mathrm{c}}\right) / U_{\mathrm{c}}$ for $U \searrow U_{\mathrm{c}}$ (respectively in $\left(\sigma-\sigma_{\mathrm{p}}\right) / \sigma_{\mathrm{p}}$ for $\left.\sigma \searrow \sigma_{\mathrm{p}}\right)$. For $U<U_{\mathrm{c}}$, on the other hand, the averaged distance is of the order of the plate distance $D$ diverging in the limit $D \rightarrow \infty$. This result is obtained by Poisson-Boltzmann theory and confirmed by computer simulations of the primitive electrolyte model with explicit micro-ions.

The physical reason for the localisation-delocalisation is as follows: for $U>U_{\mathrm{c}}$ (respectively for $\sigma<\sigma_{\mathrm{p}}$ ), all electrolyte ions will feel an external electric field attractive to the plates of opposite charge, and all ions will screen this field which results in a localised density profile decaying exponentially with the distance to the plates. On the other hand, for $U<U_{\mathrm{c}}$ (respectively for $\sigma>\sigma_{\mathrm{p}}$ ), a fraction of the electrolyte ions is sufficient to completely screen the surface charge. Hence the rest of them is free to delocalise between the plates due to entropy reasons. While the special case of only one species of ions in a globally charge-neutral system has been addressed in the literature [15], we are not aware that the transition has been discussed previously. This might be due to the fact that for typical electrolyte solutions between charged plates, one encounters the delocalised case, $\sigma>\sigma_{\mathrm{p}}$. This is different, however, for micro-electrodes where, at fixed bulk salt concentration, the area density, $\sigma$, can be drastically reduced by a confinement which is, however, still larger than the molecular length scales. Therefore, as we shall detail below, the transition is clearly detectable in micro-electrodes.

The letter is organised as follows: first we shall apply nonlinear Poisson-Boltzmann theory to extract the characteristics of the localisation-delocalisation transition analytically. We then confirm the predictions of Poisson-Boltzmann theory by Monte Carlo computer simulations with explicit micro-ions. Finally, we estimate that the transition is in principle verifiable in micro-electrodes.

In our theoretical model, we consider a system of two species of ions with charges $+q e$ and $-q e$, respectively, $(q>0)$, and microscopic hard-core diameters $d$. The ions are kept at a finite temperature $T$ in a container of a solvent with dielectric permittivity $\epsilon$ which is placed inside a capacitor. The condenser consists of two homogeneously and oppositely charged planar plates which are a distance $D$ apart. The plates are not in contact with the electrolyte to prevent electrolysis. The plate charge is $\sigma_{\mathrm{p}}$ at $z=0$ and $-\sigma_{\mathrm{p}}$ at $z=D$. The total micro-ion number density per plate area is denoted $2 \sigma$. The system is globally charge neutral. One of the basic length scales is the microscopic Bjerrum length $\lambda_{\mathrm{B}}=e^{2} / \epsilon k_{\mathrm{B}} T$ which is the typical distance where two oppositely charged ions gain a thermal energy $k_{\mathrm{B}} T$. The opposite plate charges $\pm \sigma_{\mathrm{p}}$ lead to an external electric field in the condenser, $E=4 \pi \sigma_{\mathrm{p}} / \epsilon$. Equivalently, one may see the plate charge originating from an external voltage $U$ applied with $U=E D$. The plates are infinitely large in the $x$ and $y$ directions. We address the case of large plate distances $D$, taking formally the limit $D \rightarrow \infty$ at finite $\sigma$. The key quantities to characterise localisation and delocalisation are the inhomogeneous averaged density profiles, $\rho_{+}(z)$ and $\rho_{-}(z)$ of the cations and anions. Due to symmetry, $\rho_{-}(D / 2+z)=\rho_{+}(D / 2-z)$, hence it is sufficient to consider only one species. The whole set-up is shown schematically in fig. 1 .

An analytical approach is provided by one-dimensional nonlinear Poisson-Boltzmann theory [16-18] which is a mean-field approximation. This approach incorporates entropy and electrostatics but neglects correlations of the electrolyte. Let us assume that we have a situation above the transition, $U>U_{\mathrm{c}}$, such that we are dealing with localised micro-ion density profiles 


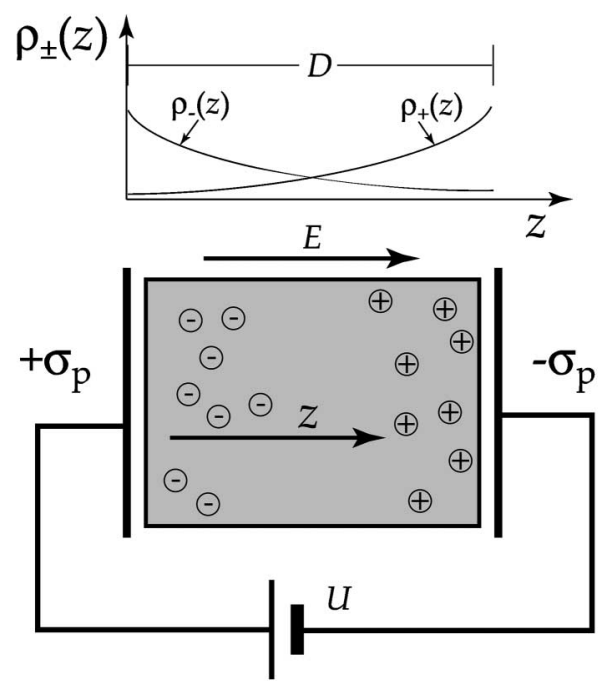

Fig. 1 - Schematic drawing of the electrolyte condenser considered. The voltage $U$ is applied across the capacitor, charging the plates to the area charge density $\sigma_{\mathrm{p}}$. This corresponds to an electric field $E$ external to the electrolyte. The microscopic cations and anions are shown in a container of dielectric fluid, and their averaged density profiles $\rho_{+}(z)$ and $\rho_{-}(z)$ are sketched as well.

close to the plates. Since the plate distance is large, it is reasonable to further assume that the cation and anion density profiles are completely separated by the midplane of the condenser at $z=D / 2$. Then Poisson's and Boltzmann's equations read as $\Phi^{\prime \prime}(z)=-4 \pi q e \rho_{-}(z) / \epsilon$, $\rho_{-}(z)=\exp \left[-q e \Phi(z) / k_{\mathrm{B}} T\right] \sigma / D$. Here, $\Phi$ is the electric potential. The analytical solution is

$$
\rho_{-}(z)=\frac{A \lambda \sigma}{\sinh ^{2}(\sqrt{A} z+\varphi)}
$$

where $A$ and $\varphi$ are determined from the boundary conditions $\int_{0}^{D} \rho_{-}(z) \mathrm{d} z=\sigma$ and $\mathrm{d} \Phi(z) /\left.\mathrm{d} z\right|_{z=0}=$ $-E$ as $\operatorname{coth} \varphi=q /(b \sqrt{A})$ and $A=[q / b-1 / \lambda]^{2}$, where $b=e /\left(2 \pi \lambda_{\mathrm{B}} \sigma_{\mathrm{p}}\right)$ is the traditional Gouy-Chapman length, and we introduce the localisation length $\lambda=1 /\left(2 \pi q^{2} \lambda_{\mathrm{B}} \sigma\right)$. Clearly, for nonzero $A$ the density profile (1) is localised. Hence, necessarily, $A$ has to vanish at a possible localisation-delocalisation transition. This yields the threshold criterion for the critical electric field $E_{\mathrm{c}}=4 \pi q e \sigma / \epsilon$ or the critical external voltage $U_{\mathrm{c}}=E_{\mathrm{c}} D$. Equivalently, this can be expressed as $\sigma_{\mathrm{p}}=q e \sigma$ or $\lambda=b / q$. Note that $E_{\mathrm{c}}$ is also equal to the maximum internal electric field that is generated by complete separation of charges in the system. In other words, the density profiles are delocalised whenever the system can compensate for the external electric field by building up an equal but opposite internal field. When the external field is too strong to be compensated for, the remaining field localises the ions around the capacitor plates.

In order to quantify the localisation-delocalisation transition, we introduce the first moment of the density profile as an order parameter defining a characteristic height $h=\int_{0}^{D} z \rho_{-}(z) \mathrm{d} z / \int_{0}^{D} \rho_{-}(z) \mathrm{d} z$. Equation (1) yields

$$
h=\lambda \ln \frac{2 \delta+1}{2 \delta},
$$




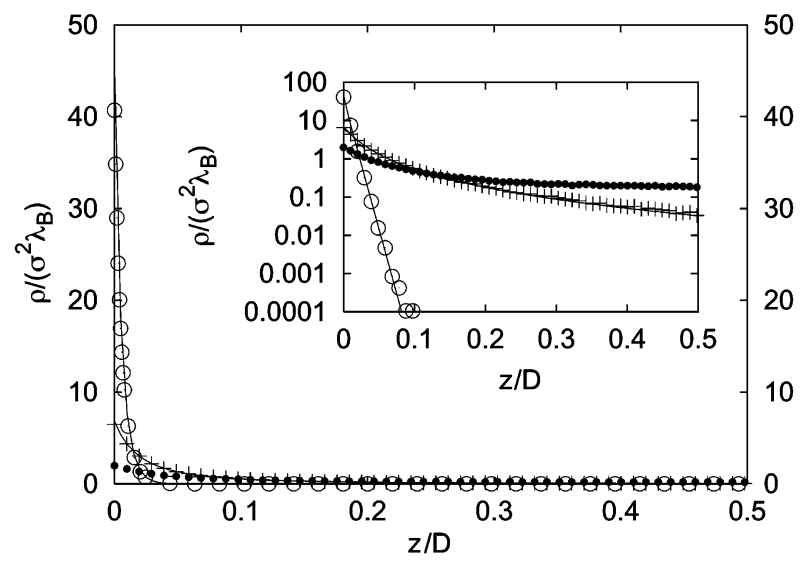

Fig. 2 - Density profiles as obtained by simulation for $\delta=3.17(\circ)$ and $\delta=0.043(+)$ and their respective theoretical predictions. A delocalised density profile $(\delta=-0.48)$ is also shown (small dots). The inset shows the same data on a semilogarithmic scale.

where $\delta=\left(U-U_{\mathrm{c}}\right) / U_{\mathrm{c}} \equiv\left(E-E_{\mathrm{c}}\right) / E_{\mathrm{c}}>0$ measures the dimensionless distance to the transition. Thus, the characteristic height, $h$, diverges logarithmically $\propto \ln \delta$ as the external voltage approaches its critical value $U_{\mathrm{c}}$.

We have further performed extensive computer simulation of the "primitive" electrolyte model where the solvent is treated as a dielectric continuum [5]. This model includes all correlations of the micro-ions which are neglected in the analytical Poisson-Boltzmann approach. We consider a finite, globally charge-neutral system of $N$ cations and $N$ anions in a rectangular simulation box with lengths $L_{x}, L_{y}, D$ and periodic boundary conditions in the $x$ and $y$ directions. Walls impenetrable for the ions are placed at $z=0, D$, and an external electric field $\boldsymbol{E}=E \boldsymbol{e}_{z}$ is applied in the $z$ direction. We take $L \equiv L_{x}=L_{y}$ and vary $D$ and $L$ to check for finite-size effects.

A Monte Carlo (MC) simulation is performed in the canonical ensemble. The Coulomb interaction is calculated via Lekner sums [19]. We use the Bjerrum length, $\lambda_{\mathrm{B}}$, as the basic length scale. The hard-core diameter of the ions is $d=0.25 \lambda_{\mathrm{B}}$ and the lateral system size is $L=512 \lambda_{\mathrm{B}}$ for most runs. Upon increasing it to $1024 \lambda_{\mathrm{B}}$ to check for finite-size effects, the results did not change. The area density per species varied between 0.95 and $4.8 \times 10^{-4} \lambda_{\mathrm{B}}^{-2}$ for the smaller system corresponding to a total number $2 N$ of simulated particles varying between 2000 and 10000 . Equilibration typically took 5000 MC steps per particle; statistics were gathered during $50000 \mathrm{MC}$ steps per particle. The distance between the capacitor plates was varied between $D=128 \lambda_{\mathrm{B}}$ to $D=4096 \lambda_{\mathrm{B}}$.

Simulation data for the density profiles for $\delta=3.17$ and $\delta=0.043$ are presented in fig. 2 . The parameters are chosen to be $\sigma=9.54 \times 10^{-4} \lambda_{\mathrm{B}}^{-2}, D=4.10 \times 10^{3} \lambda_{\mathrm{B}}$ and $L=5.12 \times 10^{2} \lambda_{\mathrm{B}}$. One can clearly see the delocalisation of the density profiles close to the transition. The predictions of Poisson-Boltzmann theory for positive $\delta$ as given by eq. (1) are also included and show favourable agreement with the simulation data.

Furthermore, we have performed an investigation very close to the transition for three different parameter combinations. The resulting heights, $h$, are shown in fig. 3 . The data is plotted using the universal scaling representation as suggested by the theory, the averaged height is scaled with the length scale $\lambda$, and the distance to the transition is just expressed by the dimensionless $\delta$. Not only do the simulation data fall onto the same universal curve, there 


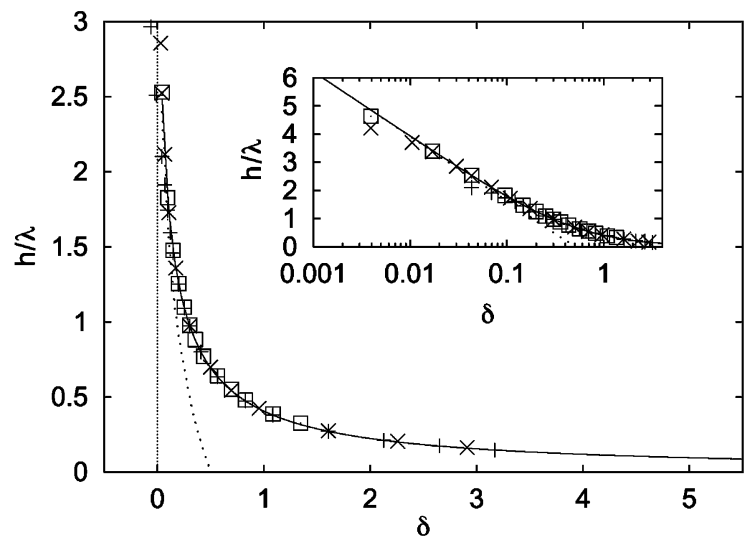

Fig. 3 - Reduced mean height, $h / \lambda$, of one species as a function of the reduced external field, $\delta$. The solid line shows the universal scaling prediction. It diverges at $\delta=0$. The asymptotic behaviour $\propto \ln \delta$ for small $\delta$ is shown as a dotted line. Simulation results are shown for different area densities, namely $\sigma=9.5 \times 10^{-4} \lambda_{\mathrm{B}}^{-2}(+), \sigma=7.6 \times 10^{-3} \lambda_{\mathrm{B}}^{-2}(\times)$, and $\sigma=1.9 \times 10^{-2} \lambda_{\mathrm{B}}^{-2}(\square)$. The other parameters were $D=4.10 \times 10^{3} \lambda_{\mathrm{B}}$ and $L_{x}=L_{y}=5.12 \times 10^{2} \lambda_{\mathrm{B}}$. The inset shows the same data in a semi-logarithmic plot.

is even quantitative agreement with the prediction (2) of our analytical Poisson-Boltzmann approach. The inset of fig. 3 shows the same data on a semi-logarithmic plot proving that there is indeed a logarithmic divergence in the simulation data. Based on both figs. 2 and 3 we conclude that the predictions of the Poisson-Boltzmann theory are quantitatively confirmed by the simulation. Regarding the divergence of $h$ close to the transition $(0<\delta \ll 1)$, we think that Poisson-Boltzmann theory is still adequate. Correlation effects may lead to micro-ion structuring and layering near the walls. Halfway between the plates, however, the concentration of micro-ions is small close to the transition such that Poisson-Boltzmann theory is still applicable there. Therefore we believe that the asymptotic form of the divergence (2) will not be changed for strong Coulomb coupling. The same insensitivity is expected for surface charge modulations $[20,21]$ provided the modulation length is much smaller than the plate spacing, $D$.

Finally, we discuss whether the condition at the delocalisation-localisation transition, $\sigma_{\mathrm{p}}=q e \sigma$, can be fulfilled in realistic samples. This criterion needs high plate charges, $\sigma_{\mathrm{p}}$, and small electrolyte densities, $\sigma$. Let us subsequently estimate limits for these two quantities. Very highly charged plates immersed into an electrolyte will lead to chemi- and physisorption of the microscopic ions [22] such that they are not any longer dissociated. A typical maximum for an effective renormalised plate charge is given by the Manning threshold value [23] and is of the order of $\sigma_{\mathrm{p}}=1 \mathrm{e} / \lambda_{\mathrm{B}}^{2}$. In aqueous solution at room temperature, monovalent electrolytes typically have bulk concentrations ranging from at least $10^{-6} \mathrm{~mol} /$ litre to $10^{-3} \mathrm{~mol} /$ litre. Depending on the electrolyte concentration, the criterion therefore requires plate distances which are in the range between a millimetre and a micrometre to see the transition. The conclusion from this estimate is that the transition can in principle be observed in small condensers. However, for realistic electrolyte concentrations, one has to go down to micro-electrodes. In order to prevent the applied voltage from driving a current through the system, which would lead to electrolysis, the capacitor plates should be mounted outside the cell. Furthermore, the cell should be placed between the plates in its entirety so that no reservoir of ions outside the electric field exists. 
In an experiment on micro-electrodes, one can see the fingerprints of the transition by watching the electrolyte concentration directly. This is in principle possible by fluorescent marking of the micro-ions (see, e.g., $[24,25]$ for recent progress in this domain). Alternatively, the transition may be observed by watching the response of the system $[26,27]$ under an AC electric field. Close to the localisation-delocalisation transition, there should be a marked anomaly in the resistance at low frequencies; a delocalised state should contribute much more efficiently to the conductivity than a localised one. Another implication of the delocalisationlocalisation transition concerns the transport of mesoscopic neutral particles with an electric dipole moment (e.g., proteins close to the isoelectric point) in the transverse direction of a micro-electrode. Tuning the external voltage such that the system parameters are close to the transition, it is expected that the effective interaction and the transport through such a microfluidic device can be tailored at wish.

In conclusion, we have predicted a sharp localisation-delocalisation transition in an electrolyte solution confined to a condenser as a function of the external voltage applied. At the transition, there is a qualitative change in the screening of the condenser plates by the electrolyte. There are either enough electrolyte ions in order to completely screen the plate charge leaving additional ions in the solution which are delocalised due to entropy. Or micro-ions are missing for complete screening, but then they are all localised. Crossing through the transition produces a logarithmic divergence of the averaged distance of the micro-ions to the plates in the limit of large plate distances. The basics of this transition is a simple competition between entropy and electrostatics, and nonlinear Poisson-Boltzmann theory describes it adequately. It would be interesting to systematically investigate electrolytes in micro-electrodes in order to detect this transition experimentally.

We finally point out that a similar effect occurs in asymmetric electrolytes and even for strongly asymmetric systems such as nanometric proteins or mesoscopic charged colloids. It would be interesting to expose sedimenting charged suspensions [28-31] to an additional electric field and study the corresponding levitation [32].

We are grateful to P. Turq, J. P. Hansen, E. Allahyarov, H. H. von Grünberg and J. F. Dufreche for helpful discussions. Financial support within the DFG, SFB TR6, project section D1, is gratefully acknowledged.

\section{REFERENCES}

[1] Gouy G., J. Phys. (Paris), IX (1910) 457.

[2] Chapman D. L., Philos. Mag., 25 (1913) 475.

[3] Parsegian V. A. and Gingell D., Biophys. J., 12 (1972) 1192.

[4] McCormack D., Carnie S. L. and Chan D. Y. C., J. Colloid Interface Sci., 169 (1995) 177.

[5] Hansen J.-P. and Löwen H., Annu. Rev. Phys. Chem., 51 (2000) 209.

[6] Netz R. R., J. Phys. Condens. Matter, 16 (2004) S2353.

[7] Meier-Koll A. A., Fleck C. C. and von Grünberg H. H., J. Phys. Condens. Matter, 16 (2004) 6041.

[8] Golestanian R., Europhys. Lett., 52 (2000) 47.

[9] Golestanian R., Europhys. Lett., 58 (2002) 712.

[10] Green N. G., Ramos A., Gonzalez A., Morgan H. and Castellanos A., Phys. Rev. E, 61 (2000) 4011.

[11] Gonzalez A., Ramos A., Green N. G., Castellanos A. and Morgan H., Phys. Rev. E, $61(2000) 4019$. 
[12] Sur U. K., Marken F., Rees N., Coles B. A., Compton R. G. and Seager R., J. Electroanal. Chem., 573 (2004) 175.

[13] Chen S. W., Ingram R. S., Hostetler M. J., Pietron J. J., Murray R. W., SchaAff T. G., Khoury J. T., Alvarez M. M. and Whetten R. L., Science, 280 (1998) 2098.

[14] Katemann B. B. and Schuhmann T., Electroanalysis, 14 (2002) 22.

[15] Lau A. and Pincus P., Eur. Phys. J. B, 10 (1999) 175.

[16] Deserno M. and Holm C., Electrostatic Effects in Soft Matter and Biophysics, edited by Holm C., KéKicheff P. and Podgornik R., Vol. 46 (Kluwer) 2001, p. 27.

[17] Andelman D., Handbook of Biological Physics, edited by Lipowsky R. and Sackmann E., Vol. 1 (Elsevier) 1995, p. 603.

[18] Carnie S. L. and Torrie G. M., Adv. Chem. Phys., 56 (1984) 141.

[19] Lekner J., Physica A, 176 (1991) 485.

[20] White T. O. and Hansen J. P., J. Phys. Condens. Matter, 14 (2002) 7649.

[21] Lukatsky D. B., Safran S. A., Lau A. W. C. and Pincus P., Europhys. Lett., 58 (2002) 785.

[22] Tellez G. and Trizac E., Phys. Rev. E, 68 (2003) 061401.

[23] Rouzina I. and Bloomfield V. A., J. Phys. Chem., 100 (1996) 9977.

[24] Callan J. F., da Silva A. P., Ferguson J., Huxley A. J. M. and O’Brien A. M., Tetrahedron, 60 (2004) 11125.

[25] Hua J. and Wang Y. G., Chem. Lett., 34 (2005) 98.

[26] Dufreche J. F., Simonin J. P. and Turq P., J. Mol. Liq., 79 (1999) 137.

[27] Dufreche J. F., Bernard O., Turq P., Mukherjee A. and Bagchi B., Phys. Rev. Lett., 88 (2002) 095902.

[28] Piazza R., Bellini T. and Degiorgio V., Phys. Rev. Lett., 71 (1993) 4267.

[29] Rasa M. and Philipse A. P., Nature, 429 (2004) 857.

[30] van Roij R., J. Phys. Condens. Matter, 15 (2003) S3569.

[31] Hynninen A.-P., van Roij R. and Dijkstra M., Europhys. Lett., 65 (2004) 719.

[32] Note that the logarithmic divergence law is qualitatively different from the ordinary divergence in a levitating solution where, when gravity $g$ goes to zero, the averaged height, $h$, diverges algebraically as $1 / \mathrm{g}$. 